\title{
Demonstration of Three-Dimensional Electrostatic Trapping of State-Selected Rydberg Atoms
}

\author{
S. D. Hogan and F. Merkt \\ Laboratorium für Physikalische Chemie, ETH Zürich, CH-8093, Switzerland
}

(Received 15 September 2007; published 30 January 2008)

\begin{abstract}
A three-dimensional trap for Rydberg atoms in selected Stark states has been realized experimentally. $\mathrm{H}$ atoms seeded in a supersonic expansion of Ar are excited to the low-field seeking $n=30, k=25,|m|=$ 0,2 Rydberg-Stark states, decelerated from a mean initial velocity of $665 \mathrm{~m} / \mathrm{s}$ to zero velocity in the laboratory frame and loaded into a three-dimensional electrostatic trap. The motion of the cold Rydberg atom cloud in the trap and the decay of the trapped atoms have been studied by pulsed electric field ionization and imaging techniques.
\end{abstract}

DOI: 10.1103/PhysRevLett.100.043001

PACS numbers: 33.80.Rv, 32.60.+i, 37.10.De, 37.20.+j

The possibility to excite atoms and molecules to Rydberg states which exhibit very large electric dipole moments presents the opportunity for efficient control of their translational motion using inhomogeneous electric fields, as was first proposed by Wing [1] and Breeden and Metcalf [2] in the early 1980s. Recently experiments have been performed in which control over the translational motion of both Rydberg atoms and molecules has been demonstrated [3-7], and Rydberg atoms in supersonic beams have been stopped [8] and trapped in two dimensions [9]. Three-dimensional (3D) and quantumstate selective trapping of Rydberg atoms or molecules is a prerequisite for many future applications in highresolution spectroscopy and quantum-information processing, and represents a major goal in this field. The present letter describes the first experimental realization of a 3D electrostatic trap for Rydberg atoms in selected Stark states. Trapping is achieved by generating an electric field minimum in 3D. The field gradients around this minimum give rise to forces that confine the Rydberg atoms in the trap.

The excitation of alkali metal atoms, accumulated in magneto-optical traps, to Rydberg states has been the principal scheme employed to produce samples of cold, quantum-state-selected Rydberg atoms and molecules up to now [10-15]. This approach has led to studies of manybody effects arising from the large dipole-dipole interactions between Rydberg atoms in thermal samples [10-13] and in Bose-Einstein condensates [14], and of the evolution from cold dense Rydberg clouds into cold plasmas $[12,15]$. However, in none of these cases are the excited Rydberg atoms trapped. 3D trapping of Rydberg atoms in states of high magnetic quantum number, $|m|$, using strong magnetic fields has been achieved starting with a laser-cooled sample of Rb atoms [16]; however, the method of production of the required high- $|m|$ states is not state selective and it has not yet been possible to trap a large number of atoms in this way. Electrodynamic trapping of ground state atoms has also been reported [17,18].

The use of pulsed supersonic beams as the starting point from which to produce cold trapped Rydberg atoms and molecules has the advantages that it leads to the formation of internally cold samples and that it is appropriate for a considerably wider range of species than only those which can be efficiently laser cooled. However, steps must be taken to first decelerate the cold atoms or molecules before loading them into a trap. The electrode arrangement presented here is designed to decelerate and trap Rydberg atoms or molecules excited to low-field seeking Stark states and is applicable to a broad range of quantum states [9]. The measurements have been performed at principal quantum number $n=30$, using $k=25,|m|=0$, 2 Stark states, where $k$ is the difference between the parabolic quantum numbers $n_{1}$ and $n_{2}$ [19]. These states exhibit an electric dipole moment of $\frac{3}{2} a_{0} e n k \simeq 2900 \mathrm{D}$, where $a_{0}$ is the Bohr radius and $e$ is the elementary charge.

The general experimental setup and excitation scheme employed in this work are similar to those reported previously [9] but rely on a newly designed electrode arrangement which enables trapping of Rydberg atoms in 3D. A supersonic beam of $\mathrm{H}$ atoms is produced by $193 \mathrm{~nm}$ excimer laser photolysis of $\mathrm{NH}_{3}$, seeded in Ar, in a quartz capillary mounted on the base plate of a pulsed valve operated at a stagnation pressure of 3.0 bar. After passing through a skimmer the beam of ground state $\mathrm{H}$ atoms enters the electrode arrangement presented in Fig. 1(a). Initially potentials of $+20 \mathrm{~V}(-20 \mathrm{~V})$ are applied to electrodes 1 and 4 (electrodes 2 and 3) to form a quadrupolar electric field distribution in the $y z$ plane with its minimum located at the midpoint of these four electrodes as shown in Fig. 1(b). Electrodes 5 and 6 are operated at potentials of $\pm 55 \mathrm{~V}$ to achieve confinement of the atom cloud in the $x$ dimension; see Fig. 1(c).

The electric field resulting from the potential difference between electrodes 5 and 6 has the effect of lifting the minimum electric field strength between electrodes 1-4 from zero to $\sim 9 \mathrm{~V} / \mathrm{cm}$ and, in doing so, ensures that a quantization axis for the state-selected Rydberg atoms exists throughout the trap volume. The resulting 3D trap is longer in the $x$ dimension than in the $y$ and $z$ dimensions [compare Figs. 1(b) and 1(c)]. The trap confines the atoms in $3 \mathrm{D}$ with the lowest saddle point lying $46 \mathrm{~V} / \mathrm{cm}$ above 
(a)

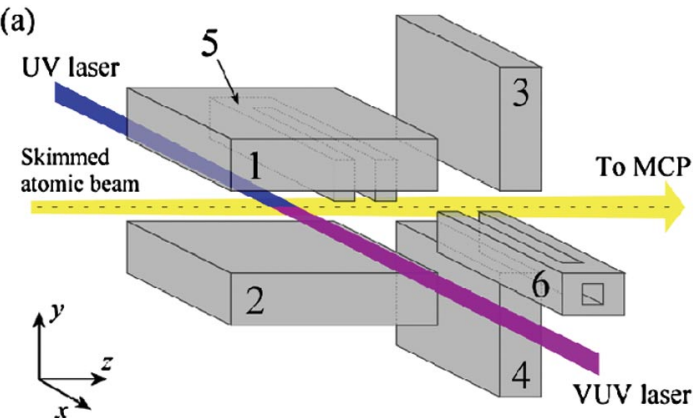

(b)

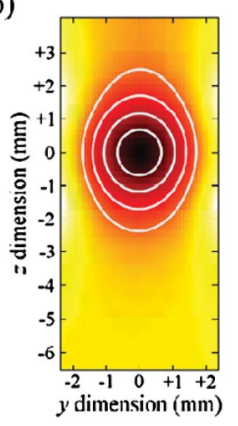

(c)

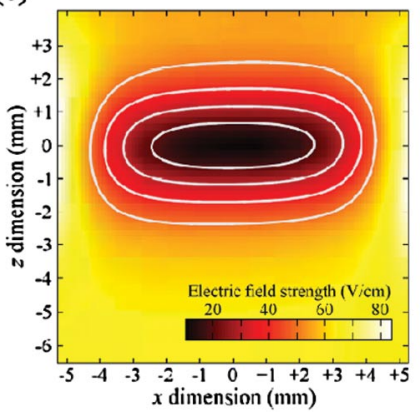

FIG. 1 (color). (a) Schematic diagram of the electrode arrangement for Rydberg-Stark deceleration and 3D trapping. The skimmed, pulsed supersonic beam of $\mathrm{H}$ atoms propagates along the positive $z$ axis. VUV and UV laser beams cross it at right angles at the midpoint between electrodes 1 and $2 . \mathrm{H}^{+}$ions produced by electric field ionization are detected at an MCP positioned on the $z$ axis $20 \mathrm{~cm}$ away from the center of the trap. The separation between electrodes 1 and 2 ( 3 and 4 ) is $6 \mathrm{~mm}$ in the $y$ dimension, that between electrodes 1 and 3 ( 2 and 4) is $3 \mathrm{~mm}$ in the $z$ dimension and that between electrodes 5 and 6 is $15 \mathrm{~mm}$ in the $x$ dimension. (b) and (c) Electric field distribution in the $y z$ and $x z$ planes at the center of the trap with potentials of $\left|V_{1,2,3,4}\right|=20 \mathrm{~V}$ and $\left|V_{5,6}\right|=55 \mathrm{~V}$. In (b) and (c) the contour lines are spaced by $10 \mathrm{~V} / \mathrm{cm}$ with the center-most corresponding to a field of $20 \mathrm{~V} / \mathrm{cm}$. The color bar indicating the field strength in (c) also holds for (b).

the $\sim 9 \mathrm{~V} / \mathrm{cm}$ minimum. This corresponds to a trap depth of $2.2 \mathrm{~cm}^{-1}$ (or $E / k_{B}=3.2 \mathrm{~K}$ ) for $n=30, k=25$ Rydberg atoms.

At the midpoint between electrodes 1 and 2 the atomic beam is crossed by counterpropagating, pulsed vacuumultraviolet (VUV) and UV laser beams which drive a resonant two-photon transition to the $k=25$ component of the $n=30$ Rydberg-Stark manifold via the $2^{2} P$ state. At the point of excitation the electric field strength is $\sim 67 \mathrm{~V} / \mathrm{cm}$. The resulting cloud of excited $\mathrm{H}$ atoms has a mean longitudinal velocity of $665 \mathrm{~m} / \mathrm{s}$ along the positive $z$ axis [see Fig. 1(a) for a definition of the axes] and a mean relative kinetic energy of $E / k_{B}=300 \mathrm{mK}$. In the $y$ dimension, perpendicular to the direction of propagation of both of the laser beams and the atomic beam, the mean kinetic energy amounts to $E / k_{B}=2.3 \mathrm{mK}$, and in the $x$ dimension it is $75 \mathrm{mK}$. The excited Rydberg atom cloud has an initial full width at half maximum of $\sim 0.8 \mathrm{~mm}$ in the $y$ and $z$ dimensions and of $\sim 6 \mathrm{~mm}$ in the $x$ dimension.

The experimental determination of these parameters has resulted from a series of investigations in which the $\mathrm{H}$ atom beam has been characterized using time-of-flight and Rydberg atom imaging techniques [7-9].

Shortly after photoexcitation, pulsed potentials of $\pm 1265 \mathrm{~V}$ are applied to electrodes 3 and 4 in order to generate a large positive electric field gradient along the $z$ axis. These potentials have rise times of $\sim 50 \mathrm{~ns}$ and decay exponentially with a $1 / e$ time of $1.9 \mu \mathrm{s}$, as described in Ref. [6]. The interaction of this electric field gradient with the electric dipole moments of the excited atoms in states with a positive Stark shift gives rise to a decelerating force on the atoms. The exponential decay of the deceleration potentials is optimized so that the atoms always interact with the maximum possible electric field gradient while never experiencing an electric field large enough to ionize them. Under these conditions the bunch of excited Rydberg atoms is decelerated to zero velocity within $\sim 5 \mu$ s of the initial rise of the deceleration potentials, having travelled a distance of only $\sim 2.0 \mathrm{~mm}$ in the $z$ dimension.

At the end of the deceleration process, the potentials on electrodes 3 and 4 have returned to their initial values of $\pm 20 \mathrm{~V}$. Two unique and essential aspects of this deceleration and trapping method are that (i) the Rydberg atom cloud is stopped exactly at the minimum of the trap with no transverse loss of atoms in the final stages of trap loading, and (ii) the atoms never traverse regions of zero electric field and therefore do not undergo randomization of $k$ and $m$.

The presence of Rydberg atoms within the trap volume is detected by field ionization using a pulsed voltage of $+2 \mathrm{kV}$ with a rise time of $50 \mathrm{~ns}$ and a duration of $100 \mathrm{~ns}$ applied to electrodes 1 and 2 . The resulting $\mathrm{H}^{+}$ions are accelerated toward a micro-channel plate (MCP) detector positioned $20 \mathrm{~cm}$ away from the trap minimum along the $z$ axis. A phosphor screen is mounted on the back of the MCP so that images of the $\mathrm{H}^{+}$ions can be recorded using a charge-coupled device (CCD) camera in parallel with ion time-of-flight (TOF) profiles.

A set of Rydberg atom trapping data representing the integrated $\mathrm{H}^{+}$ion signal recorded as a function of the time delay between the excitation of the $\mathrm{H}$ atoms to RydbergStark states and their pulsed-field ionization is presented in Fig. 2 (data set A). Initially the atoms are loaded into the trap at a density in the range $10^{6}-10^{7} \mathrm{~cm}^{-3}$. Fitting a single-exponential function to the data beyond $200 \mu \mathrm{s}$ (solid red line in Fig. 2) reveals two periods in which the rates of trap loss are quite different. At early times, up to $\sim 150 \mu \mathrm{s}$, there is a rapid loss of atoms from the trap which is strongly dependent upon the stagnation pressure at which the pulsed valve is operated [9]. At the stagnation pressure of 3.0 bar at which the data in Fig. 2 were recorded this early loss of atoms can be attributed to collisions with the argon atoms in the trailing part of the gas pulse. Beyond $200 \mu \mathrm{s}$ the exponential function fitted to the experimental data has a time constant of $135 \mu$ s which is significantly longer than that of $105 \mu$ s recorded under similar condi- 


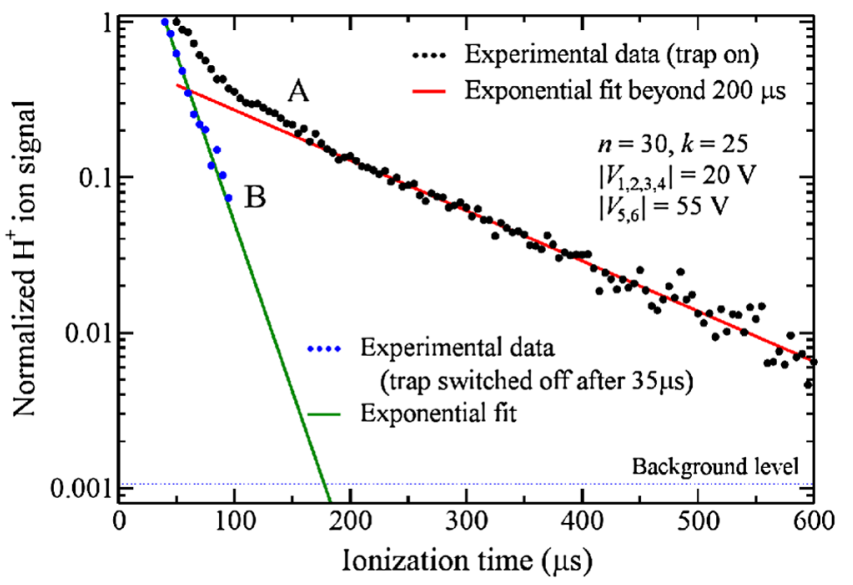

FIG. 2 (color online). Data set A: Integrated $\mathrm{H}^{+}$ion signal, indicated by $\bullet$, as a function of the time delay between excitation and pulsed-field ionization of the trapped atoms. The solid red line represents a single-exponential function with a time constant of $135 \mu \mathrm{s}$ fitted to the experimental data beyond $200 \mu \mathrm{s}$. The pulsed valve was operated at a stagnation pressure of 3.0 bar. Data set $\mathrm{B}$ : Integrated $\mathrm{H}^{+}$ion signal, indicated by $\bullet$, recorded when the trap was switched off after trapping the atoms for $35 \mu \mathrm{s}$. The solid green line represents a single-exponential function with a time constant of $20 \mu \mathrm{s}$.

tions, although with a slightly lower stagnation pressure of 2.5 bar, in previously reported $2 \mathrm{D}$ trapping experiments [9]. This trapping time constant of $135 \mu \mathrm{s}$ corresponds closely to the fluorescence lifetime of the $n=30, k=$ $25, m=0$ Stark state estimated from the values listed in Table 15 of Ref. [19]. Also included as data set B in Fig. 2 is the loss of atoms from the trap volume when the trap potentials are switched off $35 \mu$ s after loading the trap. In this case the integrated signal decays with a time constant of $\sim 20 \mu \mathrm{s}$.

In order to demonstrate full 3D trapping of the Rydberg atom cloud, the "breathing" motion of the trapped atom cloud in the $x$ dimension has been monitored by $\mathrm{H}^{+}$ion imaging. The experimental data along with simulations of the ion images are presented in Figs. 3(a) and 3(b), respectively. With potentials of $\pm 55 \mathrm{~V}$ applied to electrodes 5 and 6 , the electric field gradient experienced by the atoms located at the edges of the cloud in the $x$ dimension upon initial trap loading is large and compresses the cloud in this dimension. As seen in both the experimental and simulated images the cloud reaches its minimal size 60-70 $\mu$ s after excitation. In simulating the experimental data, the full 3D electric field distribution, calculated using a finite-element technique, is input directly along with the parameters describing the initial phase-space characteristics of the excited Rydberg atom cloud as measured experimentally [6]. The Monte Carlo particle trajectory simulations involve no free parameters but disregard collisions with the trailing part of the gas pulse and the effects of transitions induced by blackbody radiation. Each simulation presented in Fig. 3(b) corresponds to 100000 atom trajectories. The role of blackbody radiation in these experiments has been discussed in Ref. [9]. The good agreement between the experimental and simulated images validates the simulation procedure and enables one to extract precise information on the size and dynamics of the cloud of Rydberg atoms in the trap, as illustrated in Fig. 4 where the evolution of the atom cloud is presented as a function of trapping time. These data show a strong compression of the atom cloud in the $x$ dimension which leads to a minimal cloud size of $\sim 1 \mathrm{~mm}$ after $65 \mu \mathrm{s}$.

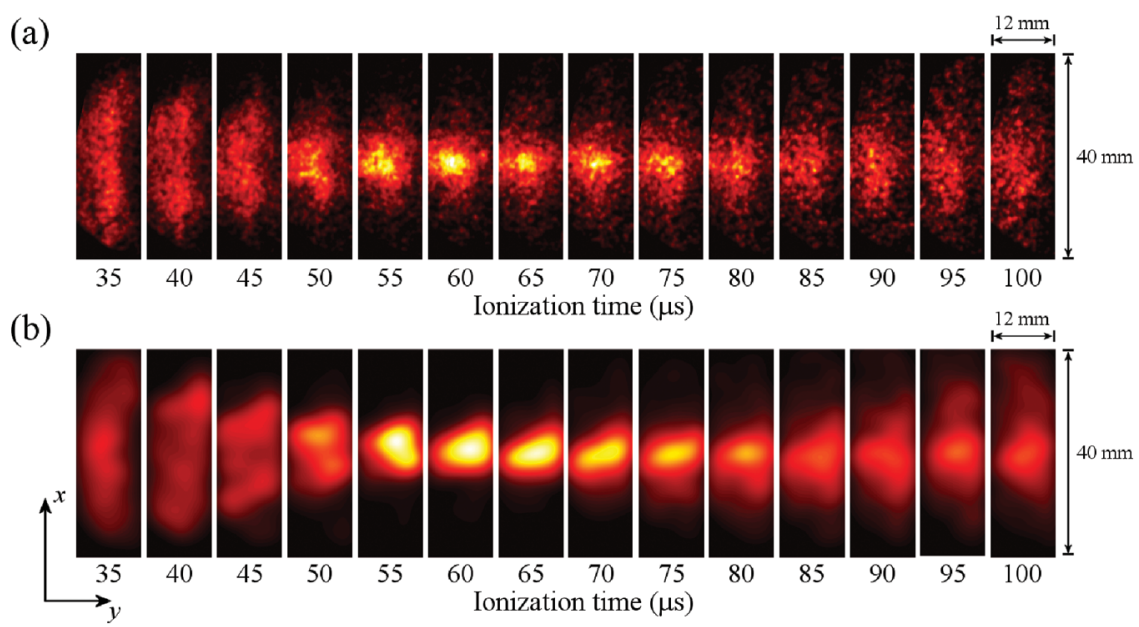

FIG. 3 (color). "Breathing" motion in the $x$ dimension of the trapped Rydberg atom cloud recorded by imaging the spatial distribution of $\mathrm{H}^{+}$ions detected at the MCP as a function of the time of pulsed-field ionization, (a) experimental data, (b) simulated data. The 3D distribution of the cloud of ions is projected onto the MCP detector which lies in the $x y$ plane. Each image is normalized by dividing by the integrated $\mathrm{H}^{+}$ion signal and the color map indicates the ion intensity normalized across each set of images. The simulated data take into account the evolution of the trapped neutral Rydberg atoms and the trajectories of the ions to the MCP detector. 


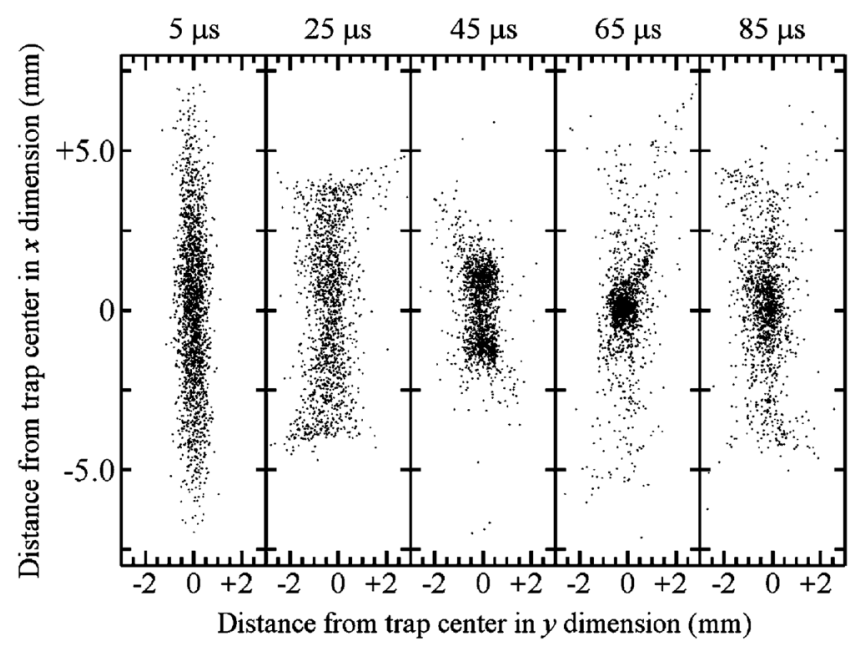

FIG. 4. Simulated spatial distributions of the Rydberg atom cloud in the $x y$ plane of the trap as a function of time. Each frame represents the spatial distribution in the trap at intervals of $20 \mu \mathrm{s}$ beginning $5 \mu$ s after excitation.

In the detection by pulsed electric field ionization, the ion times of flight recorded on the MCP are correlated to the location of the Rydberg atoms at the moment of field ionization [8]. By switching off the trap and allowing the cloud of Rydberg atoms to expand before the ionizing electric field is applied it is possible to measure the rate of expansion of the cloud of trapped atoms in the $z$ dimension. Upon switching off the trap after $100 \mu$ s the radius of the initially trapped atom cloud increases at a rate of $50 \mathrm{~m} / \mathrm{s}$ as deduced from the data presented in Fig. 5 . This corresponds to a mean kinetic energy of $E / k_{B} \sim$ $150 \mathrm{mK}$. Because of the rapid equilibration that results from the anharmonicity of the trap potential, the tempera-

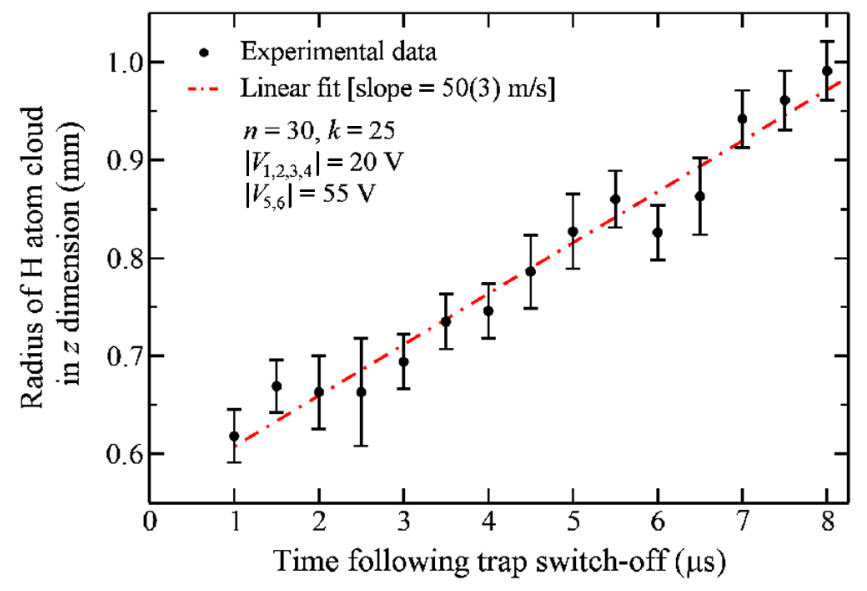

FIG. 5 (color online). Expansion of the $\mathrm{H}$ atom cloud in the $z$ dimension after switching off the trap at $100 \mu \mathrm{s}$. The radius of the cloud has been determined from the time-of-flight profiles of the $\mathrm{H}^{+}$ions to the MCP detector following electric field ionization. The dashed line is a least-squares fit to the data, the slope of which gives the expansion rate of $50(3) \mathrm{m} / \mathrm{s}$. ture measured in the $z$ dimension closely corresponds to the temperatures in the $x$ and $y$ dimensions. This rapid equilibration is also revealed by the simulations (not shown).

In conclusion full 3D electrostatic trapping of atomic hydrogen Rydberg atoms in well-defined $n$ and $k$ states has been demonstrated for the first time. The measured trap decay constant of $135 \mu \mathrm{s}$ at $n=30$ corresponds closely to the natural lifetime of the Rydberg-Stark states studied. Because fluorescence to the ground state is the dominant decay process [9] cold ground state atoms are produced during the trapping time. As all atoms and molecules possess Rydberg states, a 3D trap of the kind demonstrated here may provide a route to producing cold stationary samples of a wide variety of species including molecules. It is also of relevance to experiments on antihydrogen, the synthesis of which results in the population of Rydberg states [20].

We thank René Gunzinger for his help constructing the 3D trap, Hansjürg Schmutz for his work on the electronics and Dr. Edward Vliegen for initial help with these measurements and fruitful discussions. This work is supported by the Swiss National Science Foundation under Project No. 200021-113886.

[1] W. H. Wing, Phys. Rev. Lett. 45, 631 (1980).

[2] T. Breeden and H. Metcalf, Phys. Rev. Lett. 47, 1726 (1981).

[3] D. Townsend, A.L. Goodgame, S.R. Procter, S.R. Mackenzie, and T. P. Softley, J. Phys. B 34, 439 (2001).

[4] S. R. Procter, Y. Yamakita, F. Merkt, and T. P. Softley, Chem. Phys. Lett. 374, 667 (2003).

[5] E. Vliegen, H. J. Wörner, T. P. Softley, and F. Merkt, Phys. Rev. Lett. 92, 033005 (2004).

[6] E. Vliegen and F. Merkt, J. Phys. B 38, 1623 (2005).

[7] E. Vliegen and F. Merkt, J. Phys. B 39, L241 (2006).

[8] E. Vliegen and F. Merkt, Phys. Rev. Lett. 97, 033002 (2006).

[9] E. Vliegen, S. D. Hogan, H. Schmutz, and F. Merkt, Phys. Rev. A 76, 023405 (2007).

[10] K. Singer, M. Reetz-Lamour, T. Amthor, L. G. Marcassa, and M. Weidemüller, Phys. Rev. Lett. 93, 163001 (2004).

[11] D. Tong et al., Phys. Rev. Lett. 93, 063001 (2004).

[12] W. Li, P. J. Tanner, and T. F. Gallagher, Phys. Rev. Lett. 94, 173001 (2005).

[13] T. Vogt et al., Phys. Rev. Lett. 97, 083003 (2006).

[14] R. Heidemann et al., Phys. Rev. Lett. 99, 163601 (2007).

[15] M. P. Robinson, B. Laburthe Tolra, M. W. Noel, T. F. Gallagher, and P. Pillet, Phys. Rev. Lett. 85, 4466 (2000).

[16] J.-H. Choi, J. R. Guest, A.P. Povilus, E. Hansis, and G. Raithel, Phys. Rev. Lett. 95, 243001 (2005).

[17] S. Schlunk et al., Phys. Rev. Lett. 98, 223002 (2007).

[18] T. Rieger, P. Windpassinger, S. A. Rangwala, G. Rempe, and P. W. H. Pinkse, Phys. Rev. Lett. 99, 063001 (2007).

[19] H. A. Bethe and E.E. Salpeter, Quantum Mechanics of One- and Two-Electron Atoms (Springer, Berlin, 1957).

[20] M. Amoretti et al., Nature (London) 419, 456 (2002). 